\title{
Study of Graphene-based 2D-Heterostructure Device Fabricated by All-Dry Transfer Process
}

\author{
Dung Hoang Tien ${ }^{1}$, Jun-Young Park ${ }^{1}$, Ki Buem Kim ${ }^{1}$, Naesung Lee ${ }^{1}$, Taekjib Choi ${ }^{1}$, Philip Kim², \\ T Taniguchi ${ }^{3}, \mathrm{~K}_{\text {Watanabe }}{ }^{3}$, Yongho Seo ${ }^{1, *}$ \\ ${ }^{1}$ Faculty of Nanotechnology \& Advanced Materials, HMC, and GRI, Sejong University, Seoul \\ 143-747, South Korea \\ ${ }^{2}$ Department of Physics, Harvard University, Cambridge, MA 02138, USA \\ ${ }^{3}$ National Institute of Materials Science, Ibaraki 305-0044, Japan
}

*corresponding authors: yseo@sejong.ac.kr 


\section{Supplementary Information}

\section{How to calculate the mobility of the graphene on hBN and sandwich devices}

Electric mobility values of the fabricated devices are extracted from fitting results to the following equations.

$$
\begin{gathered}
R_{\text {tot }}=R_{\text {con }}+\frac{N}{\sqrt{n_{0}^{2}+(n)^{2}} \times 1.6 \times 10^{-19} \times \mu}, \\
\left(V_{g}-V_{D}\right)=n \frac{e}{C_{B G}}, \text { and } n=\frac{C_{B G}}{e}\left(V_{g}-V_{D}\right),
\end{gathered}
$$

where $C_{B G}$ is the geometric capacitance of the device. In case of graphene on hBN device, capacitance of the device can be considered as a series of oxide capacitance and hBN capacitance (figure S1)

$$
\frac{1}{C}=\frac{1}{C_{o x}}+\frac{1}{C_{B N}}, \quad C_{o x}=\frac{\varepsilon_{o x} \times \varepsilon_{0} \times A}{t_{o x}}, \quad C_{B N}=\frac{\varepsilon_{B N} \times \varepsilon_{0} \times A}{t_{B N}}
$$

where $\varepsilon_{o x} \approx 3.9, \varepsilon_{B N} \approx 4, t_{B N} \approx 15 \mathrm{~nm}, t_{o x} \approx 300 \mathrm{~nm}$. Therefore, the resultant capacitance $C \approx C_{o x}$, and the capacitance due to $\mathrm{hBN}$ can be ignored.
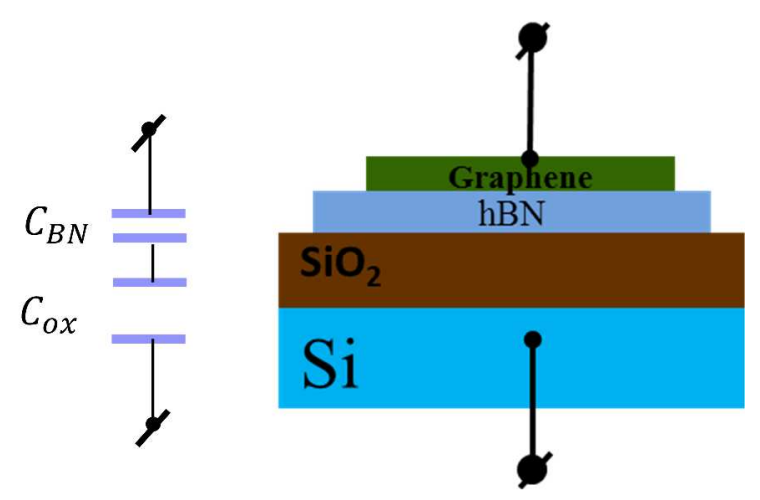

Figure S1: Schematic of capacitance of a graphene on hBN device 


\section{Geometric factor and mobility of the fabricated devices}

To calculate the mobility, the length and width of the samples were determined using optical microscope. Table S1 shows the values of the geometric factors and the mobilities of several samples.

Table S1: Geometric factor and mobility of the sandwich structures and graphene on $\mathrm{SiO}_{2}$ samples

\begin{tabular}{|c|c|c|c|c|}
\hline \multirow[t]{2}{*}{$\begin{array}{l}\text { Sample } \\
\text { number }\end{array}$} & \multirow[t]{2}{*}{ Type } & \multirow{2}{*}{$\begin{array}{l}\text { Geometry } \\
\text { factor } \\
\left(N=\frac{l}{w}\right)\end{array}$} & \multicolumn{2}{|c|}{$\begin{array}{l}\text { Mobility at room temp } \\
\left(\mathrm{cm}^{2} / \mathrm{V} . \mathrm{s}\right)\end{array}$} \\
\hline & & & in air & in vacuum \\
\hline SW\#1 & Sandwich & $\approx 6$ & 89,000 & 90,000 \\
\hline SW\#2 & Sandwich & $\approx 5$ & 100,000 & \\
\hline SW\#3 & Sandwich & $\approx 5.5$ & 68,000 & 70,000 \\
\hline SW\#4 & Sandwich & $\approx 6$ & 59,000 & 60,000 \\
\hline BN\#1 & On hBN & $\approx 10$ & 15,000 & 18,000 \\
\hline BN\#2 & On hBN & $\approx 5$ & 36,000 & 37,000 \\
\hline BN\#3 & On hBN & $\approx 3$ & 14,000 & 17,000 \\
\hline Si\#1 & On Si & $\approx 1.5$ & 1,300 & 1,500 \\
\hline $\mathrm{Si} \# 2$ & On Si & $\approx 3$ & 8,000 & 10,000 \\
\hline $\mathrm{Si \# 3}$ & On Si & $\approx 4$ & 1,500 & 3,000 \\
\hline $\mathrm{Si} \# 4$ & On $\mathrm{Si}$ & 3 & 13,000 & 15,000 \\
\hline
\end{tabular}


2. hBN exfoliated on PDMS film

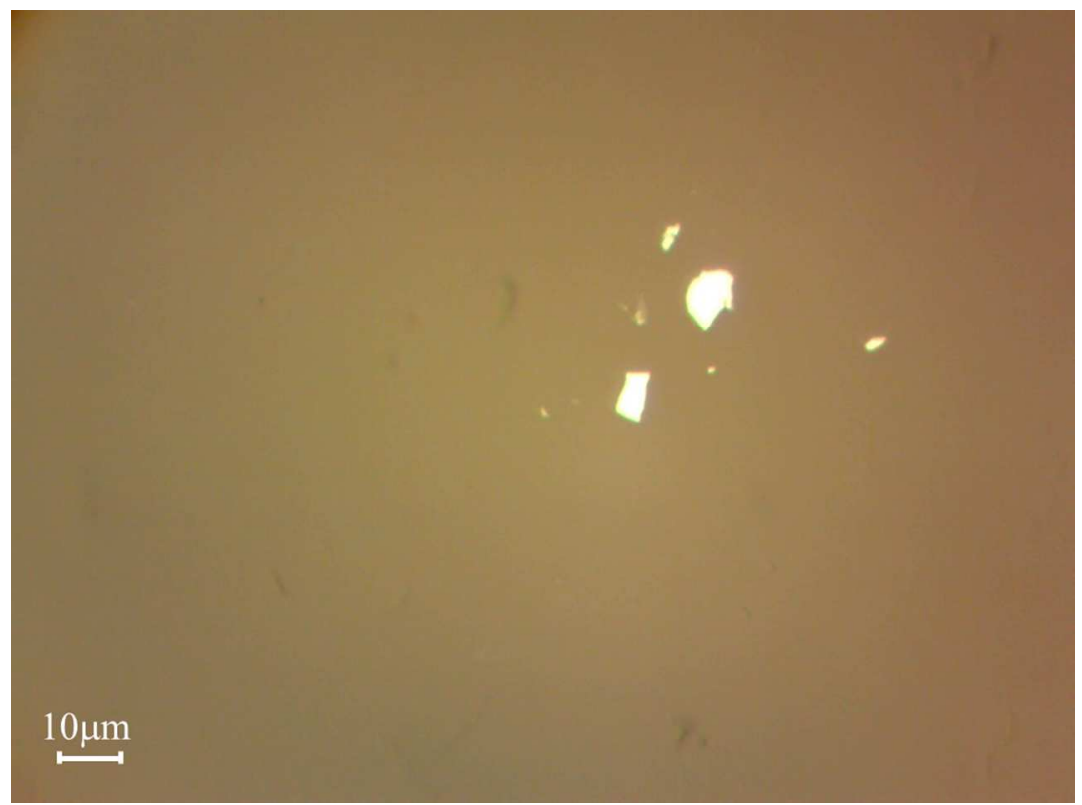

Figure S2: optical image of hBN exfoliated on PDMS film. 


\section{Anomalous behavior sandwich structured graphene in vacuum}

(a)

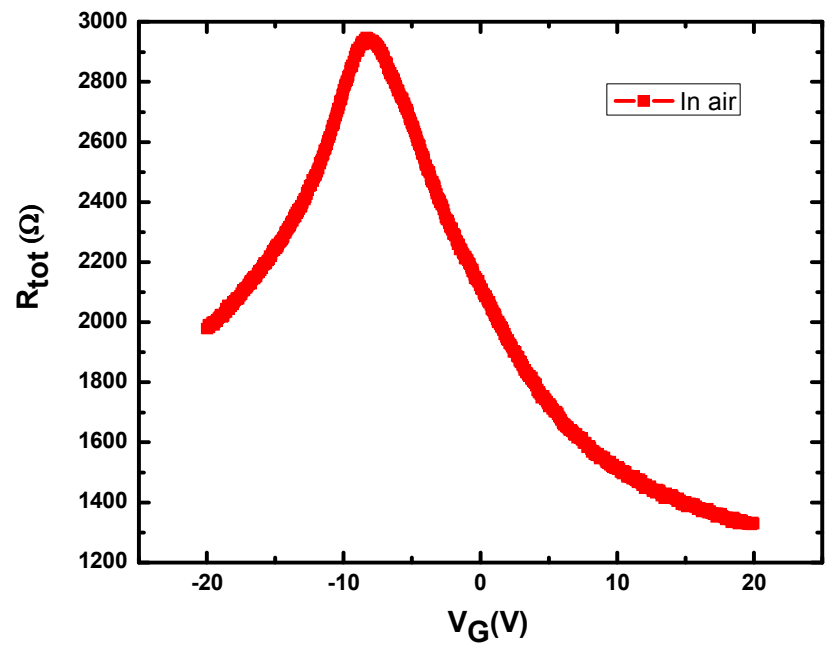

(b)

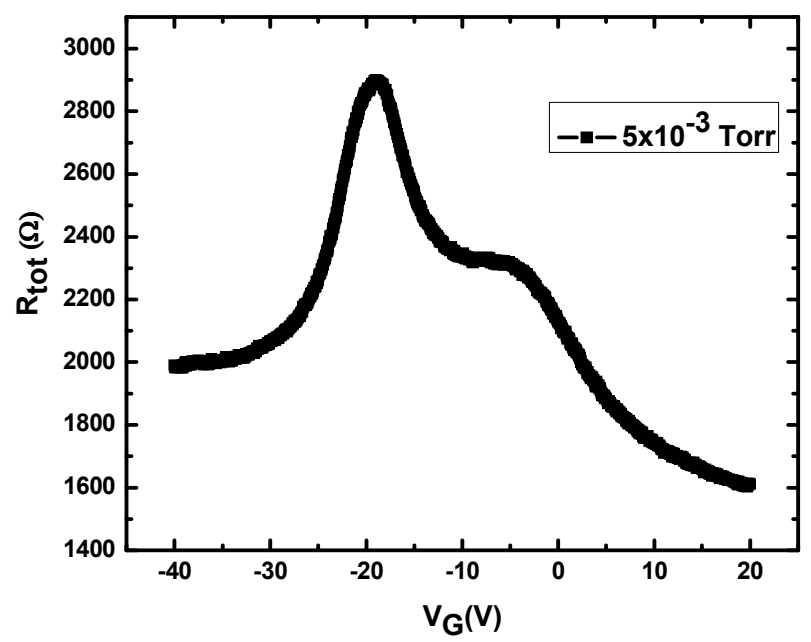

Figure S3. (a) The relationship between total resistance $\mathrm{R}_{\text {tot }}$ and back-gate bias $\mathrm{V}_{\mathrm{g}}$ of a sandwich structure device (sample SW\#5) in ambient condition at room temperature. (b) $\mathrm{R}_{\text {tot }}$ versus $\mathrm{V}_{\mathrm{g}}$ measured at low pressure $\left(5 \times 10^{-3}\right.$ Torr $)$. 
(a)

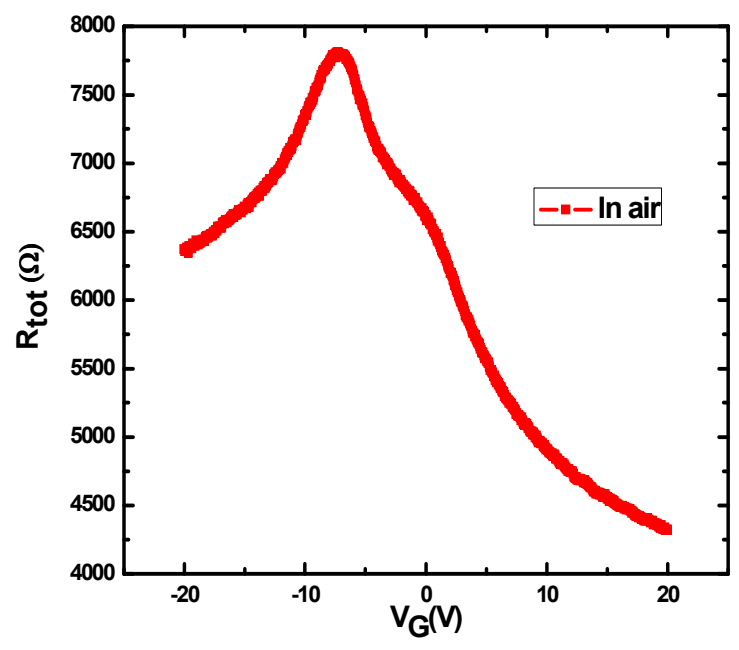

(b)

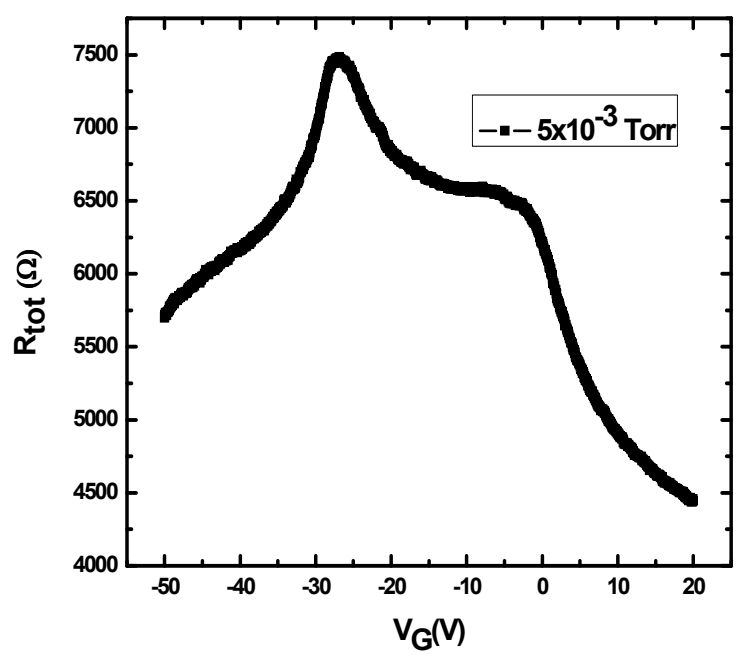

Figure S4. (a) The relationship between total resistance $\mathrm{R}_{\text {tot }}$ and back-gate bias $\mathrm{V}_{\mathrm{g}}$ of a sandwich structure device (sample SW\#6) in ambient condition at room temperature. (b) $R_{\text {tot }}$ versus $V_{g}$ measured at low pressure $\left(5 \times 10^{-3}\right.$ Torr $)$. 
(a)

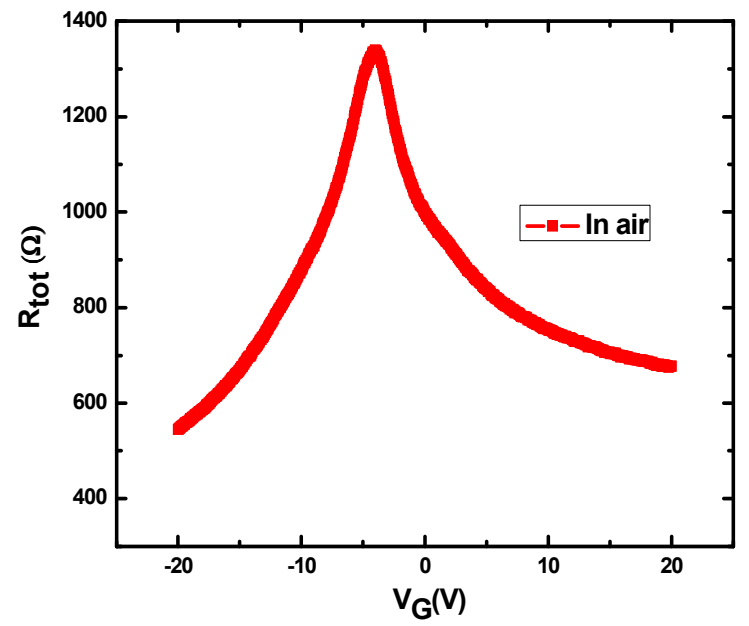

(b)

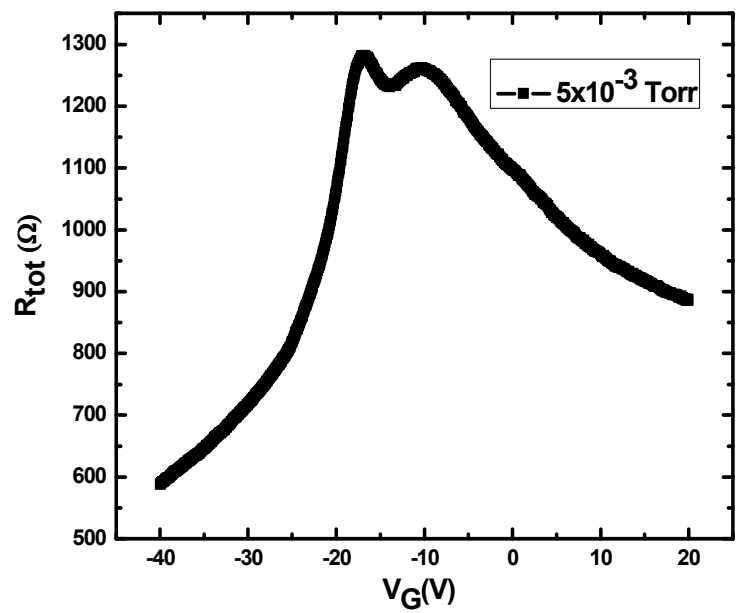

Figure S5. (a) The relationship between total resistance $R_{t o t}$ and back-gate bias $V_{g}$ of a sandwich structure device (sample SW\#7) in ambient condition at room temperature. (b) $R_{\text {tot }}$ versus $V_{g}$ measured at low pressure $\left(5 \times 10^{-3}\right.$ Torr $)$. 